\title{
A Monte Carlo framework for noncontinuous interactions between particles and classical fields
}

\author{
Christian Wesp_* Hendrik van Hees, Alex Meistrenko, and Carsten Greiner \\ Institut für theoretische Physik, Goethe-Universität Frankfurt am Main, \\ Max-von-Laue-Straße 1, 60438 Frankfurt, Germany
}

(Dated: April 24, 2022)

\begin{abstract}
Particles and fields are standard components in numerical calculations like transport simulations in nuclear physics and have well understood dynamics. Still, a common problem is the interaction between particles and fields due to their different formal description. Particle interactions are discrete, point-like events while field dynamics is described with continuous partial-differential equations of motion. A workaround is the use of effective theories like the Langevin equation with the drawback of energy conservation violation. We present a method, which allows to model noncontinuous interactions between particles and scalar fields, allowing us to simulate scatteringlike interactions which exchange discrete "quanta" of energy and momentum between fields and particles while obeying energy and momentum conservation and allowing control over interaction strengths and times. In this paper we apply this method to different model systems, starting with a simple harmonic oscillator, which is damped by losing discrete energy quanta. The second and third system consists of an oscillator and a one-dimensional field, which are damped via discrete energy loss and are coupled to a stochastic force, leading to equilibrium states which correspond to statistical Langevin-like systems. The last example is a scalar field in $(1+3)$ space-time dimensions, which is coupled to a microcanonical ensemble of particles by incorporating particle production and annihilation processes. Obeying the detailed-balance principle, the system equilibrates to thermal and chemical equilibrium with dynamical fluctuations on the fields, generated dynamically by the discrete interactions.
\end{abstract}

* cwesp@th.physik.uni-frankfurt.de 


\section{INTRODUCTION}

Monte-Carlo simulations are a mature tool in theoretical, experimental and computational physics to simulate a broad variety of problems. By randomly sampling a possible system state of a complex system ensemble, they can be applied to problems which technically do not have an immediate probabilistic interpretation [1]. In nuclear physics, MonteCarlo simulations have been used to integrate the discretized action in Lattice QCD problems [2]; in transport simulations they are used to sample collision integrals and phase-space distributions of particles [3]. Beside approaches with simple Gaussian (or white) random processes, complex and microscopic interaction kernels, based on physical properties, can be employed with help of the Boltzmann-equation [4. Hydrodynamics is another, very successful approach to the simulation of physical systems by propagating conserved quantities on a mesoscopic scale. Hydrodynamics is not directly interpreted as a classical field theory. However, its mathematical description resembles the one of fields, for example fields for the energy density and pressure (energy-momentum tensor). Both classical field theories and hydrodynamics are described by a set of deterministic, coupled partial differential equations and are generally not in the scope of Monte-Carlo methods. To implement features of noise and fluctuations, different approaches have been developed to couple random processes to the equations of motion to mimic microscopic fluctuating forces [5, 6]. This implements random processes and fluctuations in the systems but has the disadvantage of having only a statistical and long-term control over the processes. Additionally, these methods imply continuous interactions and dissipation between fields and their external forces or sources. Trying to apply those methods to particle-field interactions will bring problems as processes like particle creation and annihilation are not continuous processes.

In this paper we present a new approach which allows interactions between particles and fields without the need of effective random processes. The underlying physical interactions and processes can be modeled and simulated with Monte-Carlo methods; their impact on the system change are then propagated back to the fields and particles. This is achieved by precisely controlling momentum and energy conservation at all times of the simulation. Various examples are given in this paper, including the simulation of a thermal box with inelastic interactions between particles and fields.

This paper is organized as follows. In the first section, we summarize current methods to simulate interactions between fields and particles employing Monte-Carlo like methods and discuss their advantages and disadvantages. In section III we introduce the framework of our new method to model and simulate noncontinuous interactions between fields and particles. Sec. IV shows different examples for the implementation of our method in various test scenarios. Applications to various other physical fields and disciplines are discussed in $\mathrm{V}$. Finally, we conclude with a short summary.

\section{APPROACHES FOR PARTICLE-FIELD INTERACTIONS}

\section{A. Vlasov-Approach}

The classical Boltzmann-equation is used to describe the transport and interactions of particles in the dilute-gas limit [7]. The particles are described by a continuous distribution function which is propagated by a set of integro-differential equations. To describe charged particles in a plasma, Vlasov used the collisionless Boltzmann equation and coupled the 
particles to self-consistent field potentials [8 10] resulting in the famous (relativistic) Vlasovequation,

$$
\frac{\mathrm{d} f}{\mathrm{~d} t}=\frac{\partial f}{\partial t}+\frac{\boldsymbol{p}}{E} \cdot \frac{\partial f}{\partial \boldsymbol{x}}+\boldsymbol{F}(\boldsymbol{x}, \boldsymbol{p}, t) \cdot \frac{\partial f}{\partial \boldsymbol{p}}=0 .
$$

Eq. (1) describes the propagation of a distribution function in position and momentum space, the interesting part is the interplay between the change of momenta by the external force $\boldsymbol{F}$ and the propagation of particles by their velocity $\boldsymbol{p} / E$. The external force $\boldsymbol{F}$ can be given by an external potential and/or a mean-field interaction with the surrounding particles.

A possible way to couple a field $\phi$ with a particle distribution function $f(\boldsymbol{x}, \boldsymbol{p}, t)$ is to employ a Yukawa interaction [11]. Particles are accelerated by the gradients of the fields

$$
\boldsymbol{F}=-\nabla_{\boldsymbol{x}} E(\phi)
$$

corresponding to a mean field mean field coupling which cannot thermalize the system as it leads to reversible dynamics. By employing interactions with higher-order loops [12, 13] or by coupling the mean field to particles with collision kernels, thermalization can be recovered but on large time scales. Additionally, the interactions within the Vlasov equation are very soft and do not include inelastic processes like particle production and annihilation. Nevertheless these processes can play an important role in some applications. The linear $\sigma$-model, for example, shows a shift in thermodynamic properties when particle-number changing processes are neglected, and in dynamical calculations the chiral phase transition is lost if the field can not dissipate energy by particle production and annihilation upon a temperature change [14].

\section{B. Momentum Space Approach}

A common approach to approximate quantum field systems is to assume spatial isotropy and reduce the phase-space distribution of the physical problem to functions in momentum space. This ansatz has been very successful and answered many questions regarding non-equilibrium phenomena and thermalization [15]17]. Additionally, in quantum field theory interactions and their propagators are often well-defined in momentum space, leading to transition probabilities for single Fourier modes in terms of scattering-matrix elements, $S_{f i}=\left\langle\boldsymbol{p}_{1}^{\prime} \boldsymbol{p}_{2}^{\prime} \ldots|S| \boldsymbol{p}_{1} \boldsymbol{p}_{2} \ldots\right\rangle$. These probabilities can be treated perturbatively, like in quantum electrodynamics (QED). In numerical simulations, such dynamics can be applied to a classical field, and the impact on the system by changing single Fourier modes is easily calculated through the sum of the modes' energies. This method works well for systems in momentum space with the assumption of spatial isotropy. In the case of spatial anisotropy or if the system propagation has to be described in position space and only the interactions are performed in momentum space, then this method implies a violation of causality. The change of a single mode in momentum space changes the field distribution instantaneously in position space over the whole volume, resulting in superluminal signal propagation. These artifacts render this method unsuitable for simulations which rely on position-space calculations. 


\section{Langevin Equation}

The Langevin or Ito-Langevin equation [18] is a stochastic differential equation, used to describe systems with two different scales. The "macroscopic" long-range and slow-timescale part is described by deterministic equations of motion. Additionally it is coupled to the "microscopic" small scales, which are described by short-ranged fast random processes. For a heavy particle in a heat bath, the original Langevin equations reads

$$
m \ddot{x}(t)=-\gamma \dot{x}(t)+F_{\text {ext }}(x, t)+\xi(t)
$$

with an external force $F_{\text {ext }}$, the linear damping coefficient $\gamma$, and a stochastic force $\xi$. Often a simple Gaussian white-noise process is assumed for the fluctuating force, which leads to an average energy flux from and into the bulk system, while the damping is the dissipative average part with the back reaction of the medium to the energy-momentum exchange neglected.

In nuclear physics, the Langevin Equation has been applied on top of the Boltzmann equation to include fluctuations in the system [19]22]. By dividing dynamics of a scalar quantum field in a hard and a soft part, a stochastic description of the system can be employed which resembles a Langevin equation [23]. The Langevin equation can be used to investigate fluctuations in the linear $\sigma$-model [24] or with similar methods to investigate disoriented chiral condensates [25]. Using the influence formalism, classical equations for the $O(N)$ fields at presence of a heat bath can be derived, when a stochastic interpretation is employed [26]. In [27 29] the Langevin equation has then been employed to phenomenologically model a stochastic coupling of a locally equilibrated (hydrodynamical) particle bath and a classical field within a linear $\sigma$-model. This coupling allows an effective thermalization of the mean field.

However, the Langevin equation has some drawbacks, the dissipation of the equation (3) due to the friction term, $\gamma \mathrm{d} \phi(t) / \mathrm{d} t$ is a continuous process. This is a natural assumption for continuous systems like fields or waves and a reasonable approximation for systems with a clear separation of scales, like in the classical example of a heavy particle in a bath of small ones. However, many processes are discrete and occur as single events. The same problem holds for the random force, $\xi$, which acts continuously and changes its value with every time step in numerical implementations. Because of the random nature of this process, the exact amount of exchanged energy can only be controlled in a statistical manner, and the back reaction of the bulk medium is neglected. In most implementations, the random force $\xi(t)$ is modeled by Gaussian white noise without a memory kernel. Using a more sophisticated ansatz with memory kernel, the random force can be extended to a non-Markovian stochastic process with colored noise [30].

Before we discuss the relation between momentum and energy dissipation within a Langevin equation, we have to define them for a field $\phi$. For a general Klein-Gordon equation,

$$
\partial_{\mu} \partial^{\mu} \phi+m^{2} \phi+\frac{\partial U}{\partial \phi}=0
$$


the following conserved quantities can be defined [31, 32]:

$$
\begin{aligned}
E & =\int_{V} \mathrm{~d}^{3} x \epsilon(\boldsymbol{x}) \\
& =\int_{V} \mathrm{~d}^{3} x\left[\frac{1}{2} \dot{\phi}^{2}+\frac{1}{2}(\vec{\nabla} \phi)^{2}+U(\phi)\right], \\
\boldsymbol{P} & =\int_{V} \mathrm{~d}^{3} x \boldsymbol{\Pi}(\boldsymbol{x})=\int_{V} \mathrm{~d}^{3} x \dot{\phi} \vec{\nabla} \phi,
\end{aligned}
$$

where $E$ denotes the total field energy, and $P$ is the total momentum. For any positivedefinite potential $U$, the relation

$$
P \leq E
$$

holds. The dissipative part of the Langevin equation for the field $\gamma \partial_{t} \phi$ damps both the energy (5) and the momentum (6). For a potential-free wave equation with damping,

$$
\partial_{t}^{2} \phi(t, x)+\gamma \dot{\phi}(t, x)=\nabla^{2} \phi(t, x)
$$

the ratio of $P(t) / E(t)$ is non-linear in time because both quantities are non-linear operators while $\dot{\phi}$ is linear. This results in different damping rates for $E$ and $P$ and complicates any attempt to couple particles and fields through inelastic interactions within an effective model.

Another problem arises with the continuous nature of the dissipative term in the Langevin equation. For a continuous process, quantities like energy transfer can be calculated by integrating over a time interval, but this leads to a continuous value which can not be interpreted by an integer number of events. In contrast, singular events like particle-pair production and annihilation can be counted, and rates are defined in a statistical manner. This becomes a problem when one tries to couple a scalar field to an ensemble of particles with interactions given by pair production and annihilation. Energy loss of the scalar field leads to energy gain in the particle ensemble and vice versa. Such an ansatz is used in the famous and successful cosmological inflation model [33], in which particles are created by the energy loss of the oscillating scalar field, $\Phi$. Particle production is described by rate equations, which are derived from the fields' equations of motion. Trying to simulate such a process with finite ensembles of particles leads to different problems. The energy loss within a time step $\Delta t$ can be calculated from the fields and mapped to a certain number of created particles. The energy of a discrete number of created particles will, however, never match exactly with the continuous loss rate. Additionally, the physical process of pair production will depend on the simulation time-step size, and for $\Delta t \rightarrow 0$ a mapping between the continuous dissipation and the noncontinuous particle creation will not be possible anymore. Another problem is the fact that the random force $\xi(t)$ changes its value at every point in time, both for white and colored noise. Trying to couple this behavior to pair production and annihilation leads to the same problem as the microscopic processes will depend on the time step.

In summary, the Langevin equation is a very good choice for an effective description of a system with two separate time scales. However, a microscopic modeling of the interaction processes is complicated by the continuous nature of the Langevin process. In the next section we will present how to potentially solve these problems by a noncontinuous approach. 


\section{NONCONTINUOUS INTERACTIONS BETWEEN FIELDS AND PARTI- CLES}

This work is originally motivated by the need to describe inelastic and discrete interactions between fields and particles. The process of creating particles and anti-particles from field excitations and vice versa,

$$
\bar{q}+q \leftrightarrow \phi
$$

turns out to be very important for a consistent description of chemical and thermal equilibration in the linear $\sigma$-model and in our transport simulation DSLAM (Dynamical Simulation of a Linear SigmA Model) [14. The particles are modeled with the test-particle ansatz, and the fields are represented by classical scalar fields. Any interactions beyond mean-field couplings have to be discrete, because energy and momentum can only be transferred in terms of whole particle pairs; we therefore have developed a particle-field method which allows such noncontinuous interactions. The method is split up in two parts: a framework for transferring an exact amount of energy and momentum from and to a field and a field theoretical calculation for taking possible interactions between fields and particles into account in a microscopic way. The second method enables us to develop Monte-Carlo models for the interaction probability of a given process, and the first method allows us to realize the interactions in a simulation.

\section{A. Discrete energy and momentum transfers from and to a field}

Particles and fields are described in a very different manner. While scalar fields define a single quantity as function of time and position in continuous space (or discrete space on a grid), particles are characterized by their position and momentum. For particles, this sums up to three position coordinates, the energy and three-momentum values at every point in time. Fields are described by continuous functions or $N^{3}$ values on a three-dimensional $N$-sized grid for the field $\phi(\boldsymbol{x}, t)$ and its time derivative, $\dot{\phi}(\boldsymbol{x}, t)$.

To link these very different descriptions, common mathematical and physical properties have to be found. The most simple approach is to use energy and momentum. For particles, energy and momentum are directly given by their momentum four-vector. For a field we can employ the already discussed relations (5) and (6). A discrete interaction now maps to a discrete change of energy and momentum at a given position $\boldsymbol{x}$ and time $t_{k}$. The field $\phi\left(\boldsymbol{x}, t_{k}\right)$ is propagated with its undisturbed equations of motion and changed due to an interaction by a kick $\delta \phi\left(\boldsymbol{x}, t_{k}\right)$ which changes the energy and momentum by the desired amount $\Delta E$ and $\Delta \boldsymbol{P}$. This leads to relations of four coupled non-linear equations,

$$
\begin{aligned}
\Delta E\left(t_{k}\right) & =E\left[\phi\left(\boldsymbol{x}, t_{k}\right)+\delta \phi\left(\boldsymbol{x}, t_{k}\right)\right]-E\left[\phi\left(\boldsymbol{x}, t_{k}\right)\right], \\
\Delta \boldsymbol{P}\left(t_{k}\right) & =\boldsymbol{P}\left[\phi\left(\boldsymbol{x}, t_{k}\right)+\delta \phi\left(\boldsymbol{x}, t_{k}\right)\right]-\boldsymbol{P}\left[\phi\left(\boldsymbol{x}, t_{k}\right)\right] .
\end{aligned}
$$

In general (10) and (11) have to be solved with a numerical non-linear equation solver. For $\Delta E<0$ energy will be taken out of the field, $\Delta E>0$ will add this amount energy (and analogously for the momentum).

Without further constraints, Eqs. (10) and (11) have either no solutions or infinitely many. To define and find unique solutions, the disturbance kick $\delta \phi\left(\boldsymbol{x}, t_{k}\right)$ has to be parameterized. In general, $\delta \phi\left(\boldsymbol{x}, t_{k}\right)$ must have a finite support to keep causality. Furthermore it can not be a point like disturbance as this will cause instabilities in the field equations as well as 


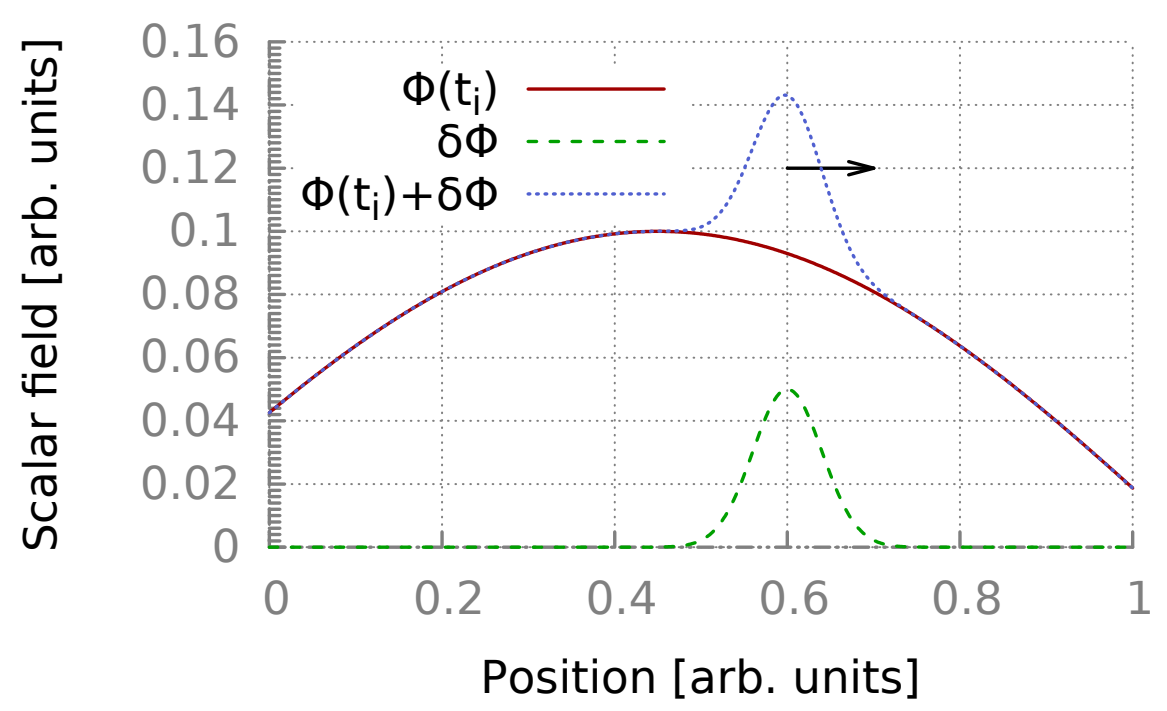

FIG. 1. (Color online) Visualization of the interaction principle, as described in (12). The energy of the initial field $\phi(t)$ is changed by a parameterized field variation $\delta \phi$. The resulting field $\phi(t)+\delta \phi$ is increased by a given energy $\Delta E$ and momentum $\Delta \boldsymbol{P}$. The traveling direction of the Gaussian is depending on the momentum.

numerical problems [14]. Therefore, the parameterization should be as smooth as possible to avoid shocks and numerical artifacts on the scalar field.

A useful and robust parameterization is a three-dimensional, moving Gaussian wave packet,

$$
\delta \phi(\boldsymbol{x}, \boldsymbol{v})=\left.A_{0} \prod_{i}^{3} \exp \left[-\frac{\left(x_{i}-v_{i} \tilde{t}\right)^{2}}{2 \sigma_{i}^{2}}\right]\right|_{\tilde{t} \rightarrow 0},
$$

where $\boldsymbol{v}$ defines the velocity of the Gaussian wave packet, $A_{0}$ the strength of the interaction, and the parameter $\tilde{t}$ is needed to define and calculate the derivatives for energy and momentum in (5) and (6). The three position arguments $x_{i}$ are fixed by the interaction position.

To find $A_{0}$ and $\boldsymbol{v}$ in the parameterization (12), which solve (10) and (11) for a given $\Delta E$ and $\Delta \boldsymbol{P}$, the four coupled and non-linear equations have to be solved with a numerical equation solver with $\Delta E$ and $\Delta \boldsymbol{P}$ given by the physical interaction; the definition of these quantities will be given in various examples in the following Sections. A simple visualization of this principle is given in Figure 1, which shows the local modification of the field by a Gaussian.

The three widths of the Gaussian, $\sigma_{i}$, are free parameters and can be fixed to a single spherical radius by $\sigma_{x}=\sigma_{y}=\sigma_{z}=\sigma$. It determines the interaction volume and should be chosen to fit the system scale. It has an impact on the minimal scale of possible modes in the system, as we will see in (47).

Furthermore, the parameterization $(12)$ should be seen as a non-relativistic approximation which is valid for small velocities. The parameterization is not Lorentz invariant, resulting in spatial extent which does not depend on the velocity. This has the interesting effect, that for a $(1+3)$ D system the maximal momentum to energy ratio which can be generated with 12. is $\max \left\{\frac{P}{E}\right\}=1 / 2$. The parameterization can be extended with a Lorentz boost, for 
example along the $x$-direction for $\boldsymbol{v}=\left(v_{x}, 0,0\right)$

$$
\begin{aligned}
\delta \phi(\boldsymbol{x}, t)= & A_{0} \exp \left(-\frac{\gamma^{2}\left(x-v_{x} t\right)^{2}}{2 \sigma^{2}}\right) \\
& \times \exp \left(-\frac{y^{2}+z^{2}}{2 \sigma^{2}}\right)
\end{aligned}
$$

The Lorentz boost leads to a disc-shaped deformation of the initially spherical Gaussian. With the boost, the momentum to energy ratio of (13) has the correct relativistic limit.

$$
\lim _{v \rightarrow 1}\left\{\frac{P}{E}\right\}=1
$$

At $v=0.3$ both solutions differ by a factor of about $18 \%$, for small velocities they are nearly the same, and $(12)$ can be used as a safe approximation.

\section{B. Modeling of energy transfers}

In Sect. III A, the mathematical framework for energy and momentum transfer has been discussed. To apply this method to physical systems, additional modeling has to be done. The above method describes, how to transfer a given amount of energy $\Delta E$ and momentum $\Delta \boldsymbol{P}$ to or from a scalar field at a given interaction point $\boldsymbol{x}$ and time $t_{i}$. To realize this method in a transport or Monte-Carlo simulation, appropriate values for $\Delta E, \Delta \boldsymbol{P}, \boldsymbol{x}$ and $t_{i}$ have to be defined. Motivated by the stochastic interpretation of interaction cross sections, a probability distribution for energy and momentum exchanges,

$$
P(\Delta E, \Delta \boldsymbol{P}, \Delta t),
$$

can be defined, which has to be derived according to the physical model; in Sect. IV] we will give various examples, calculations and results for such a modeling. In general, the probability distribution (15) can have a memory kernel and can depend on the whole history with $P(\Delta E, \Delta \boldsymbol{P}, t)$, but we will use the Markov approximation in which only the current system state is important for future events, and (15) depends on the time-step size, $\Delta t$, only.

\section{EXAMPLES AND MODEL CALCULATIONS}

\section{A. Discretely damped harmonic oscillator}

As the most simple test system, we choose the classical one-dimensional oscillator with damping,

$$
\ddot{x}(t)+\gamma \dot{x}(t)+\omega_{0}^{2} x=0 .
$$

In this example the mass of the system is set to unity. With no loss of generality units like $c, \hbar$ and $k_{B}$ are set to unity in the rest of the paper, and physical quantities like the energy are chosen to have an arbitrary unit (arb. units).

The frictional part $\gamma \dot{x}$ dissipates energy from the system in a continuous process. We want to model the same system to have a discrete, noncontinuous damping such that, within an 
ensemble average, both systems should behave the same. We assume to have a weak damping of the oscillator with $\gamma<\omega_{0}$. The analytic solution of the oscillator's energy scales with

$$
E(t) \approx \bar{E}_{0} \mathrm{e}^{-\gamma t}
$$

So the linear damping term $\dot{x}$ leads to an exponential energy loss in the oscillator. To model a discrete damping for (16), we could introduce a deterministic formalism which removes a given quantum of energy at fixed times out of the system. The second and more natural choice is a probabilistic ansatz, which models the system's initial total energy $E_{0}$ as a sum of small energy quanta $\overline{\Delta E}$,

$$
E_{0}=N_{0} \cdot \overline{\Delta E},
$$

where $N_{0}$ is the initial number of energy quanta and can also be called "steppiness" because it defines in which energy steps the system can be damped. The energy of the system can be damped by changing the number of energy quanta $N(t)$

$$
E(t)=N(t) \cdot \overline{\Delta E}
$$

This change of $N(t)$ has to be modeled according to the equations of motion. We now explain how to find an interaction-probability function like 15 for this system. In this example, we can assume a two-state interaction: for a given $\Delta t$, the oscillator can lose a quantum of energy $\overline{\Delta E}$, or it can be left undisturbed. For $\Delta t \ll 1$ we can neglect multiple decays; additionally we assume a Markov process, so the oscillator only depends on its current state and has no "memory". Using these constraints, the interaction-probability distribution $P(\Delta E, \Delta t)$ without memory kernel can be described as

$$
P(\Delta E, \Delta t)=\underset{\text { loss }}{\operatorname{Pr}}(\Delta t) \delta(\Delta E-\overline{\Delta E})+\underset{0}{\operatorname{Pr}}(\Delta t) \delta(\Delta E)
$$

with $\operatorname{Pr}_{\text {loss }}$ being the probability to lose an energy quantum $\Delta E$ in the time interval $\Delta t$, while $\mathrm{Pr}_{0}$ is the probability for the system to stay unchanged. Both probabilities are related by the normalization of the probability distribution,

$$
\int P(\Delta E, \Delta t) \mathrm{d} \Delta E=\underset{\text { loss }}{\operatorname{Pr}}(\Delta t)+\underset{0}{\operatorname{Pr}}(\Delta t)=1 .
$$

To find the probability for the oscillator to lose a certain amount of energy, we assume that every energy quantum $\overline{\Delta E}$ can decay independently. The definition for the exponential decay is

$$
\frac{\mathrm{d} N(t)}{\mathrm{d} t}=-\gamma N(t)
$$

with each decay event having a constant and independent decay probability in a time step $\mathrm{d} t$ of $\operatorname{Pr}=\gamma \mathrm{d} t$. With $\mathrm{d} t \rightarrow \Delta t$ and $\Delta t \ll 1$ we can write

$$
\Delta N(t)=-\gamma \Delta t N(t)
$$

However, we want to calculate the probability of a single energy quantum to decay. The number of energy quanta is given by 19 .

$$
N(t)=E(t) / \overline{\Delta E}
$$

which increases the number of energy quanta if $\overline{\Delta E}$ decreased. 
The total probability of a decay of a single quantum in a system of many quanta in firstorder approximation factors to the product of an individual quantum's decay probability times the number of individual quanta,

$$
\underset{\text { loss }}{\operatorname{Pr}}(\Delta E)=\gamma \cdot \Delta t \cdot N(t)=\gamma \cdot \Delta t \frac{E(t)}{\overline{\Delta E}} .
$$

For $N_{0} \rightarrow \infty$ or $\Delta E \rightarrow 0$ this is the definition of the exponential decay law, while a finite $N_{0}$ will give a discrete exponential decay for a finite ensemble. For the total probabilitydistribution function we obtain

$$
\begin{aligned}
P(\Delta E, \Delta t)= & \delta(\Delta E-\overline{\Delta E})\left(\gamma \cdot \Delta t \frac{E(t)}{\overline{\Delta E}}\right) \\
& +\delta(\Delta E)\left(1-\gamma \cdot \Delta t \frac{E(t)}{\overline{\Delta E}}\right)
\end{aligned}
$$

Simulating $P(\Delta E, \Delta t)$ will give the same average energy loss scaling for $E(t)$ as the original harmonic oscillator with continuous damping.

In a numerical realization, the oscillator is propagated with the free equation of motion,

$$
\frac{\mathrm{d}^{2} x}{\mathrm{~d} t^{2}}+\omega_{0}^{2} x=0
$$

This equation of motion conserves the total energy. To simulate damping, at every time step the decay-probability density $P(\Delta E)$ is sampled using Monte-Carlo techniques. In case of a decay, the oscillator will lose the given amount $\Delta E$ by employing the method described in section III A. In case of an oscillator, only the energy equation (10) has to be solved. The change $\delta x$ on $x(t)$ becomes a simple shift of the oscillator,

$$
x_{t} \rightarrow x_{t}+\delta x \text {. }
$$

For a harmonic potential, this can be done analytically by solving

$$
\begin{aligned}
\overline{\Delta E} & =E\left(x_{t+1}\right)-E\left(x_{t}\right) \\
& =\frac{1}{2}\left[\omega_{0}^{2} x_{t+1}^{2}+\dot{x}_{t+1}^{2}-\omega_{0}^{2} x_{t}^{2}-\dot{x}_{t}^{2}\right] .
\end{aligned}
$$

The derivatives are approximated by the first-order difference discretization and with $\mathrm{d} t \rightarrow$ $\Delta t$ :

$$
\dot{x}_{t+1}=\frac{x_{t+1}-x_{t}}{\Delta t}
$$

Solving $(29)$ for $x_{t+1}$ results in a rather lengthy equation which can be simplified by neglecting all terms of order $\mathcal{O}\left(\omega^{2} \Delta t^{2}\right)$ and higher. The result is

$$
x_{t+1}=x_{t} \pm \Delta t \sqrt{2 \overline{\Delta E}+\dot{x}_{t}^{2}} .
$$

This can be seen as an addition to the undisturbed equations of motion. With $\overline{\Delta E} \rightarrow 0$ equation (31) becomes the usual, first order Euler propagation for a differential equation: $x_{t+1}=x_{t}+\Delta t \cdot \dot{x}_{t}$. The additional term $\overline{\Delta E}$ is the change of the system given by the "interaction kick", changing the total system's energy with exactly this amount of energy. 


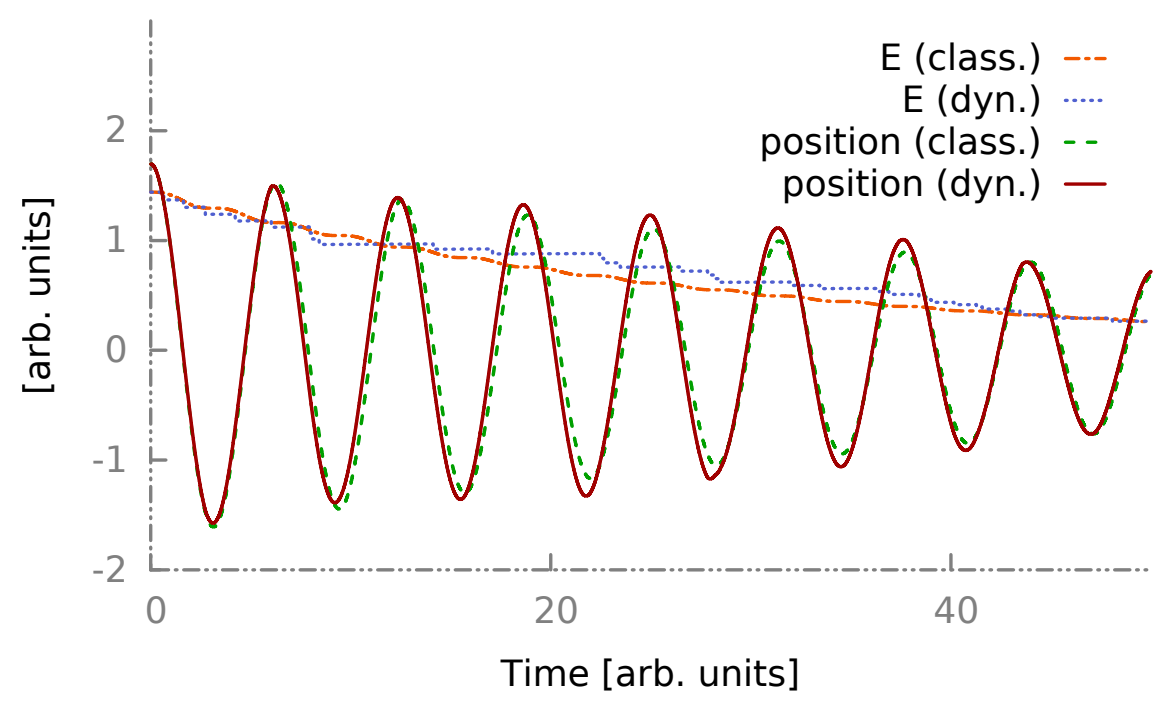

FIG. 2. (Color online) Simulation of a discretely damped harmonic oscillator. The number of simulated particles and energy excitations is $N=150$. With every decay of a particle, the oscillator loses a bit of energy, which leads to damping of its motion. The discrete and continuous version have the same ensemble average. In a single run statistical fluctuations occur.

The sign \pm in front of the square root is determined by the direction of $\dot{x}_{t}$, a kick with $\overline{\Delta E}>0$ should always point in the direction of the current velocity $\dot{x}_{t}$. Note that $\overline{\Delta E}$ can always be positive while it can only be negative if $\dot{x}_{t}^{2}-2|\overline{\Delta E}|>0$ to have a real solution for the propagation equation.

Fig. 2 shows a single simulation run of such a system. Both curves show statistical fluctuations but are in good agreement over the runtime. Averaged over many realizations, the energy shows the expected exponential decay. Using different values for $N$, the 'steppiness' can be controlled from a very smooth damping to a rough damping with only a few steps. On average, all show the exponential decay with the expected rate.

\section{B. Harmonic oscillator coupled to a heat bath (Langevin approach)}

In a second benchmark, we extend the damped harmonic oscillator by a coupling to a heat bath via a Langevin equation, similar to [34]. This calculation is done for a simple harmonic oscillator as well as for a scalar field in $(1+1)$ space-time dimensions. Again, the mass of the system is set to unity, and energy units labeled as arbitrary unit (arb. units). For the non-relativistic case, the equation of motion reads

$$
\frac{\mathrm{d}^{2}}{\mathrm{~d} t^{2}} x(t)+\gamma \frac{\mathrm{d}}{\mathrm{d} t} x(t)+x(t)=\kappa \xi(t)
$$

where $\xi(t)$ is defined as Gaussian white noise with $\left\langle\xi(t) \xi\left(t^{\prime}\right)\right\rangle=\delta\left(t-t^{\prime}\right)$, using the equipartition theorem and the fluctuation-dissipation theorem [35], we can fix the strength of the stochastic force in the equilibrium case as

$$
\kappa=\sqrt{2 \gamma T}
$$

This equation of motion describes a damped harmonic oscillator, driven by a Gaussiandistributed random force $\xi(t)$, which can increase or decrease the energy of the system by 
"kicking" the oscillator. On average, the oscillator will show a Gaussian position distribution, and by using the Fokker-Planck equation one can derive the equilibrium distribution for the energy, which is a Boltzmann distribution

$$
f(E) \propto \exp (-E / T) .
$$

To simulate the oscillator with the Langevin-dynamics within our proposed method, we propagate the system with the interaction-free equation of motion,

$$
\frac{\mathrm{d}^{2}}{\mathrm{~d} t^{2}} x(t)+x(t)=0
$$

and model the discrete interactions again as small kicks to have the same statistical averages as the original Langevin equation of motion (32). A representation for the stochastic force $\kappa \cdot \xi(t)$ in terms of energy changes can be found by using the energy-work theorem, applied to the fluctuating part of the force in the Langevin equation,

$$
\frac{\mathrm{d} E}{\mathrm{~d} t}=\dot{x}(t) \cdot F(t)=\dot{x}(t) \cdot \kappa \cdot \xi(t),
$$

which after discretization with $\mathrm{d} t \rightarrow \Delta t$ and $t \rightarrow t_{n}$ becomes

$$
\Delta E=\dot{x}(t) \cdot \Delta t \cdot \kappa \cdot \tilde{\xi}\left(t_{n}\right)
$$

The stochastic force $\tilde{\xi}(t)$ is still a Gaussian white noise with a normally distributed random number $\xi_{n}$ at each time step $t_{n}$,

$$
\tilde{\xi}\left(t_{n}\right)=\frac{\xi_{n}}{\sqrt{\Delta t}}
$$

The factor $\Delta t^{-\frac{1}{2}}$ is needed to fix the norm of the uncorrelated white noise via

$$
\left\langle\xi_{n} \xi_{m}\right\rangle=\frac{\delta_{m n}}{\Delta t}
$$

The total interaction-probability distribution function, $P(\Delta E, \Delta t)$, is now composed of four components of single probabilities. The probability of no interaction in a time interval $\operatorname{Pr}_{0}$, the damping of the oscillator by the process $\gamma \dot{x}(t)$ with the probability $\operatorname{Pr}_{\text {loss }}$, as discussed in the previous section, and the two cases where the energy of the system is changed by the stochastic force $\tilde{\xi}(t)$ : we can see in (37) that depending on the signs of $\xi$ and $\dot{x}(t)$ a random kick can add energy to a system or dissipate energy from it. Both processes are symmetric in general and from this symmetry one obtains $\langle\Delta E\rangle=0$ in equilibrium. However $\left\langle\Delta E^{2}\right\rangle>0$ is always given.

The probability $\operatorname{Pr}_{\text {loss }}$ has already been discussed in the previous section IVA and follows the same schematics here. Due to their symmetry, the loss and gain terms induced by the stochastic force, given by (37), can be subsumed in a single probability density term. The sum of all terms for the interaction probability distribution is

$$
\begin{aligned}
P(\Delta E, \Delta t)= & \delta(\Delta E-\dot{x}(t) \cdot \Delta t \cdot \kappa \cdot \xi(t)) \\
& +\delta(\Delta E-\overline{\Delta E})\left(\frac{\gamma \cdot \Delta t}{\overline{\Delta E}} E(t)\right)+\operatorname{Pr}_{0} \delta(\Delta E) .
\end{aligned}
$$


The no-interaction probability $\mathrm{Pr}_{0}$ is again fixed by the normalization condition, $\int P(\Delta E, \Delta t) \mathrm{d} \Delta E=1$. Equation (40) looks quite complicated, but the single terms can be easily interpreted and simulated. The last term is the contribution for no interaction to happen at all. The second last term describes the probability for a system to lose a given amount of energy $\overline{\Delta E}$ due to friction, just like in our first example. Finally, the first term describes the process of changing the energy from a kick by the stochastic force. The system gains energy, if the direction of the kick points in direction if the velocity, $\dot{x}(t) \cdot \xi(t)>0$, and loses energy if the kick reduces the velocity, which is the case for $\dot{x}(t) \cdot \xi(t)<0$.

To sample the gain and loss terms by the random kicks in the probability density, one can simply sample a random kick $\xi_{n}$ and calculate the given energy difference from (37), which is propagated back to the system. As we simulate a simple $(0+1) \mathrm{D}$ problem, the already known relation for changing the energy (31) can be used.

In this example we describe the dissipative process with discrete decay steps while the energy fluctuations given by $\xi(t)$ can have continuous values. Even though this seems contradictory, it has two reasons: we wanted to stay as close as possible to the original Langevin equation, which has continuous interactions. The second reason is that we wanted to introduce continuous values for $\Delta E$ at this point because in the last example in this paper (IVD) particles and fields exchange energy by discrete particle annihilation and creation processes. While these processes are discrete in time, their energy and momentum spectrum is continuous.

Fig. 3 shows the position and velocity distribution of the harmonic oscillator, which is a Gaussian. The width of the distribution depends on the temperature in the fluctuationdissipation relation (33) and scales with $\sqrt{T}$. The energy distribution shows an exponential tail $\exp (-E / T)$ with the same temperature. The numerical calculation was performed with our method, which does not solve exactly the classical Langevin equation, but a set of equations which have the same statistical description as explained above. As a result, the statistical properties of the simulated system are in good agreement due to the consistent description of the energy-exchange rates.

\section{One-dimensional scalar field coupled to Langevin equation}

The previous calculations in this work have dealt with a simple harmonic oscillator. In the next example, the scalar system (16) is advanced to a $1+1$-dimensional case with a spatially extended field. The position coordinate of the oscillator, $x(t)$, is now generalized to a scalar field $\phi(x, t)$. The equation of motion of a potential-free Klein-Gordon equation with a stochastic force and damping is given by

$$
\left(\frac{\partial^{2}}{\partial t^{2}}-\frac{\partial^{2}}{\partial x^{2}}\right) \phi(x, t)+\gamma \frac{\partial}{\partial t} \phi(x, t)+\phi(x, t)=\kappa \xi(x, t)
$$

The stochastic force is the $1+1$-dimensional extension of the Gaussian white noise $\left\langle\xi(x, t) \xi\left(x^{\prime}, t^{\prime}\right)\right\rangle=\delta\left(x-x^{\prime}\right) \delta\left(t-t^{\prime}\right)$. The stochastic process (41) is simulated as a reference system. In comparison, in our simulation the field $\phi(x, t)$ is propagated with the disturbance-free equations of motion. The stochastic force and damping are simulated analogously as in the $(0+1)$-dimensional example. Because of the additional spatial dimension of the system, we could model a momentum exchange $\Delta P$, as given in (15). We neglect this term because the original Langevin-equation does not have this term either. However, the last example in this paper will address this issue. 

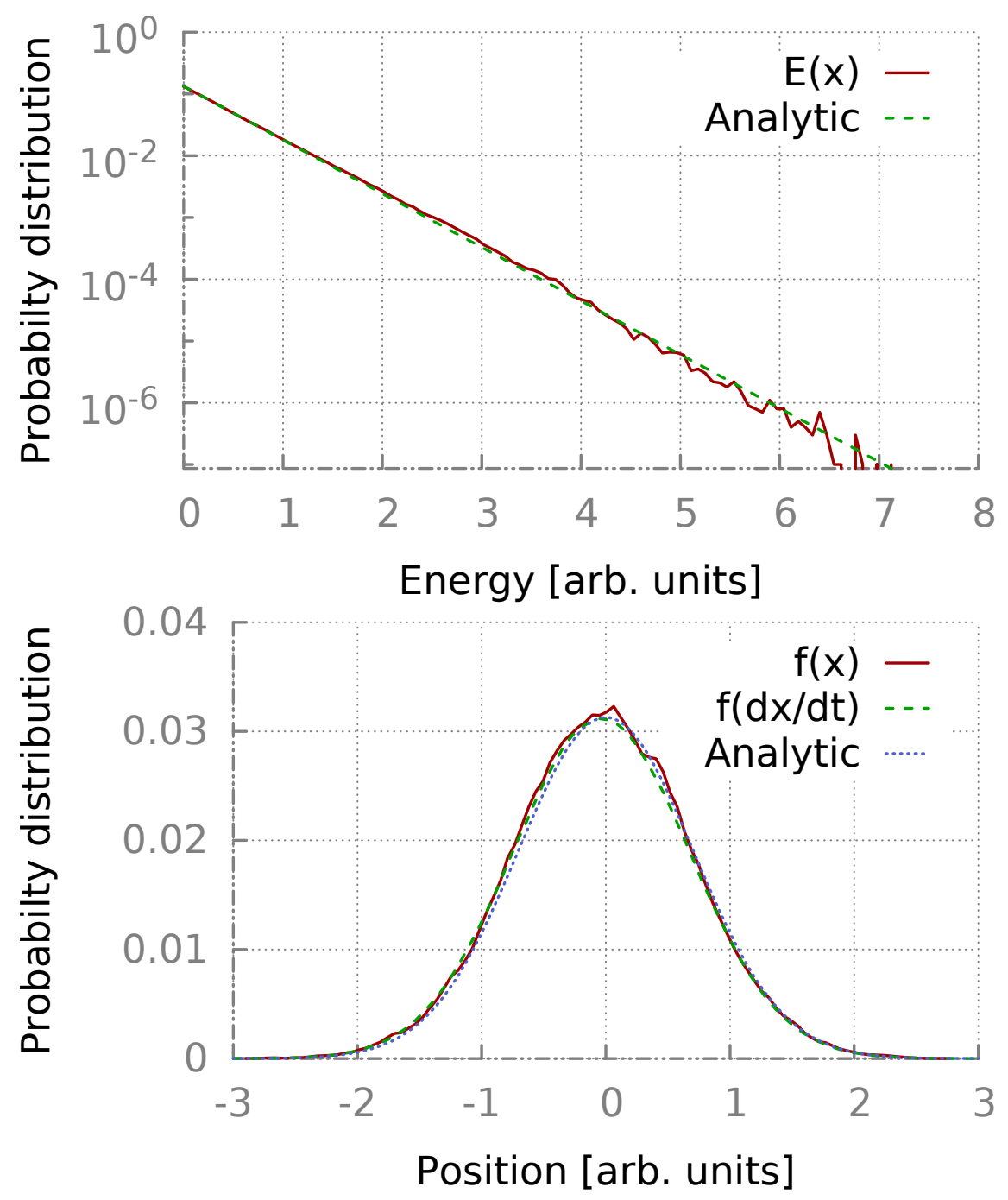

FIG. 3. (Color online) Simulation of a simple harmonic oscillator, coupled to a heat bath via a Langevin equation. The random force and the damping is implemented with the noncontinuous energy-transfer method. The plot shows the distribution function of the energy, the position distribution and the velocity distribution for a temperature of $T=0.5$ arb. units and $10^{8}$ calculation steps and a corresponding energy-step size. Both plots coincide with the expected analytical result, which is $\exp (-E / T)$ for the energy distribution (top) and $1 /(\sqrt{2 \pi T}) \exp \left(-m \omega_{0}^{2} x^{2} / 2 T\right)$ for the spatial distribution.

In the previous example of the simple oscillator, the change of the oscillator's position $\Delta x$ could be calculated with the analytic relation (31), which included both the kick from the energy exchange and the propagation by the equation of motion. Such an analytic relation can not be found anymore in this example because the spatial extent of the system and the interaction region leads to a complex dependency of the energy equation (10), requiring a numerical solution of the problem. The numerical problem decomposes in finding a solution for the kick by the change of energy and then for propagating the newly found fieldconfiguration with the equation of motion. Additionally, the change of the field $\Delta \phi(\boldsymbol{x})$ has to be parameterized, as many possible solutions could be found for eq. (10) for systems with 


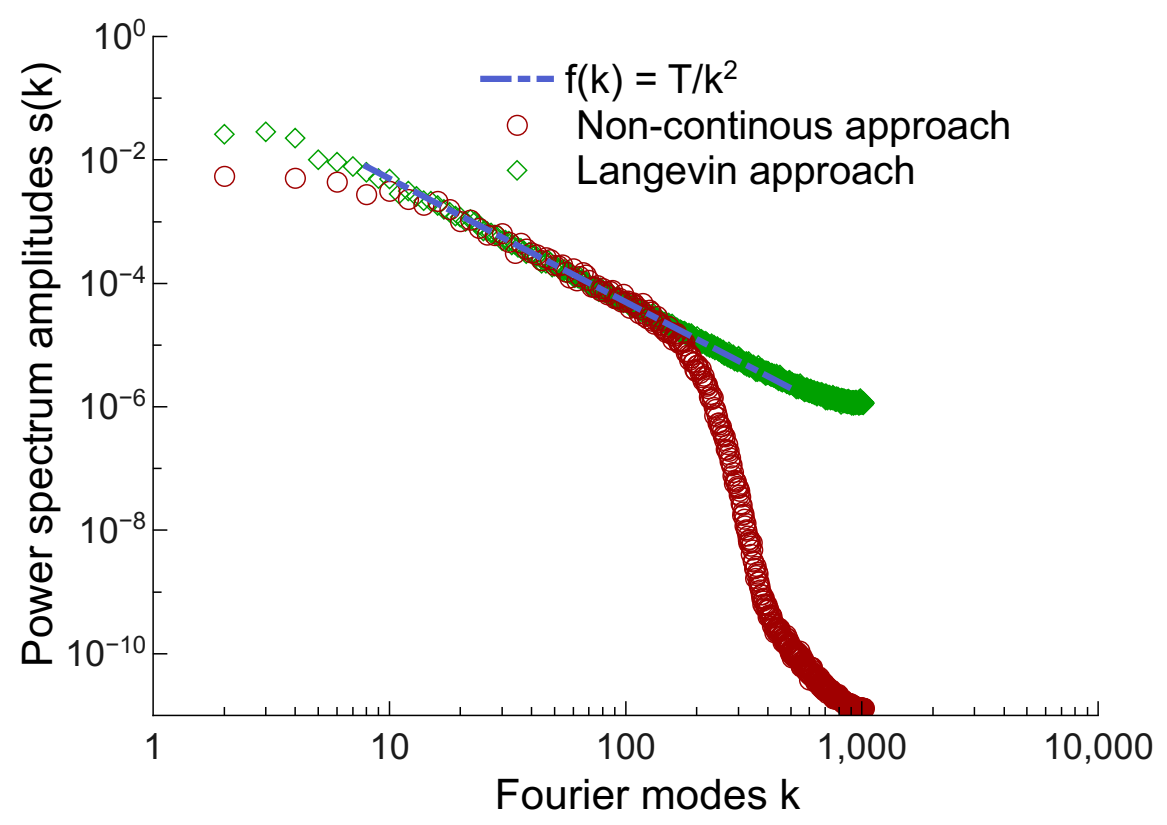

FIG. 4. (Color online) Time averaged power-spectrum of the scalar field $\phi(x, t)$ in $(1+1)$ spacetime dimensions. The spectrum is calculated according to 43 . The system is a one-dimensional harmonic oscillator, coupled to a Langevin equation with a continuous and a noncontinuous ansatz. The dashed, blue line shows the theoretical solution of the system $T / k^{2}$, the red circles the noncontinuous approach and the green diamonds show the classical Langevin equation as a reference. All three curves are in very good agreement in the intermediate region and show the same system temperature, which was chosen to $T=0.5$ arb. units Both Fourier plots are averaged over 100 snapshots of the same run. Simulated was a grid with 1024 points. At large wavelengths, both systems deviate due to finite system size effects. At small wavelengths the noncontinuous methods has an interesting cut-off due to the finite-size of the interaction region in comparison to the point-like interactions of the classical Langevin method.

a spatial extend. In this example a one-dimensional form of the Gaussian parameterization (12) has been chosen.

For each point of the system an interaction probability is sampled with (40). In case of an interaction, the center of the Gaussian interaction parameterization is located at the interaction point, and the change of the field $\phi(\boldsymbol{x}, t)+\delta \phi(\boldsymbol{x})$ is solved by using equation (10) with help of a numerical solver. For every interaction at some point $\boldsymbol{x}$ the neighbor cells of the interaction point are changed as well. The reason is the spatial extension of the Gaussian parameterization leading to a smeared interaction region or volume. This holds both for adding energy as well as removing energy from the system.

As shown in figure 1, this will result in a field given by a superposition of small interaction "bumps". In the equilibrium state, the field distribution will show spatial fluctuations, distributed among the field's modes. Overall the equilibrium state behaves very similar to the oscillator case but with a spatial extent in one dimension and therefore more dynamics. The same holds true for the interaction probability-distribution density, which is the spatially 
extended version of $(40)$ :

$$
\begin{array}{r}
P(x, \Delta E, \Delta t)=\delta(\Delta E-\dot{\phi}(x, t) \cdot \Delta t \cdot \kappa \cdot \xi(x, t)) \\
\quad+\delta(\Delta E-\overline{\Delta E})\left(\frac{\gamma \cdot \Delta t}{\bar{\Delta} E} E(x, t)\right)+\operatorname{Pr}_{0} \delta(\Delta E) .
\end{array}
$$

A very interesting observable is the power spectrum of the field,

$$
S(\boldsymbol{k})=\lim _{t \rightarrow \infty} \frac{1}{2 t} \int_{-t}^{t} \mathrm{~d} t|\mathcal{F}[\phi(x, t)](\boldsymbol{k})|^{2}
$$

with the spatial Fourier transformation $\mathcal{F}$

$$
\mathcal{F}[\phi](\boldsymbol{k}, t)=\int \mathrm{d}^{3} \boldsymbol{x} \exp (-i \boldsymbol{k} \cdot \boldsymbol{x}) \phi(\boldsymbol{x}, t) .
$$

In case of the classical Langevin equation, the expected distribution of the power spectrum can be calculated. A damped field coupled to a white-noise process is expected to show Brownian noise, as it effectively integrates the white noise over time [36]. The resulting field has an average power spectrum with the temperature as the mode amplitude [35, 37],

$$
S(k)=\frac{T}{k^{2}} .
$$

Fig. 4 shows the expected spatial spectrum for the classical Langevin equation (41) and for the simulation with our proposed method. For large and small momenta $k$, deviations occur due to finite-system effects, in the intermediate region the spectrum shows a very good agreement with (45). At some point the small wavelengths with large $k$ are suddenly strongly suppressed.

The explanation for this behavior is the fact that we use a finite volume excitation in the parameterization (12). In a classical Langevin equation, the point-like stochastic force $\xi$ has a constant spectrum $S_{\xi}(k) \sim c$, allowing to excite any modes. Within our method, the energy is changed in a small but finite-size sub-volume. The smallest excitable mode in the system has therefore the same scale as the interaction volume. Within the Gaussian parameterization (12), this scale is the width $\sigma$. The resulting mode cutoff can be calculated evaluating the spectrum of the parameterization $(12)$, here for our $(1+1)$ dimensional example:

$$
|\mathcal{F}[\delta \phi(x, t)](k)|^{2} \sim e^{-k^{2} \cdot \sigma^{2} / 2} \approx \begin{cases}1 & \text { for } k \ll \sigma, \\ 0 & \text { for } k \gg \sigma\end{cases}
$$

At the scale $k^{2} \sigma^{2} \approx 1$ the Gaussian shape leads to a soft cutoff which suppresses all higher modes, preventing the well-known UV catastrophe of classical thermal fields. We therefore define a soft cutoff scale at

$$
k_{\text {Cutoff }} \equiv \frac{\sqrt{2}}{\sigma} \text {. }
$$

These results show that our method is capable of simulating a thermal system with Langevin dynamics with controlled systematic numerical errors. 


\section{Particle ensemble coupled to scalar field}

In this Section we use the proposed method to couple an ensemble of particles to a scalar field in a $(3+1)$-dimensional simulation. The simulated system is a microcanonical box with a scalar field, particles and anti-particles. Particles can perform two-body elastic collisions, the field is propagated via a wave equation, like in the sections before. Additionally, field and particles can interact by microscopic particle annihilation and creation processes. This system is motivated by a physical problem which is discussed in an upcoming paper. However, we want to use these calculations to demonstrate the proposed method in a more complex example and try to keep the details of the used model as generic as possible, while still being exact in our derivations. The underlying physical system is the linear sigma model with constituent quarks. We model quark-pair creation and annihilation to study non-equilibrium effects near and at the phase transition. The process of creating particles from field modes and vice versa is crucial for describing critical phenomena and fluctuations driven by the dynamics of the phase transition.

The equations of motion in a general form are

$$
\begin{aligned}
\left(\frac{\partial^{2}}{\partial t^{2}}-\nabla_{\boldsymbol{x}}^{2}\right) \phi(\boldsymbol{x}, t)= & \lambda_{1} \phi^{3}(\boldsymbol{x}, t)+\lambda_{2} \phi(\boldsymbol{x}, t) \\
& +U_{\bar{q} q}(\boldsymbol{x}, t)+U_{0}
\end{aligned}
$$

with the coupling strength for the potentials $\lambda_{1}, \lambda_{2} \gg 1$, a source term $U_{0}$ and a mean field potential between the field and the particles $U_{\bar{q} q}$. Besides the mean-field and potential interactions, a particle $q$ and an anti-particle $\bar{q}$ can annihilate to a field-quantum. The underlying process is given by the Yukawa coupling,

$$
\mathcal{L}_{\text {int }}=g \bar{\sigma} \phi^{*} \phi,
$$

which can create a $\sigma$ particle by pair annihilation, $\bar{q}+q \rightarrow \sigma$, or destroy it in the inverse decay process, $\sigma \rightarrow \bar{q}+q$.

The interaction between particles and the field is modeled in several steps. The quarkannihilation process is calculated with a microscopic cross section, the generated particle is treated as an unstable particle resonance, the $\sigma$ particle. The created $\sigma$ particle is not propagated or added to the system as a real particle, but its energy and momentum are transferred to the scalar field, keeping the system's total energy and momentum conserved. In the inverse process, excitations in the scalar field are treated as energy excitations. These excitations are modeled as unstable particles which can decay to a quark-antiquark pair.

The interaction probability of the two incoming particles for the process $\bar{q} q \rightarrow \sigma$ is calculated microscopically. In our simulation it is modeled with a constant, isotropic cross section $\hat{\sigma}_{\bar{q} q \rightarrow \sigma}$ with respect to all kinematic constraints. For Monte-Carlo sampling, we use the stochastic interpretation of the cross section [4] for a set of particles in a cell employing a constant and isotropic cross section:

$$
\operatorname{Pr}(\bar{q} q \rightarrow \sigma)=\hat{\sigma}_{\bar{q} q \rightarrow \sigma} v_{\text {rel }} \frac{\Delta t}{\Delta V N_{\text {test }}}
$$

with the particles' relative velocity,

$$
v_{\text {rel }}=\frac{s}{2 E_{1} E_{2}},
$$


and the Mandelstam variable,

$$
s=\left(p_{\mu}^{1}+p_{\mu}^{2}\right)^{2} .
$$

We have chosen the Breit-Wigner cross section [38, 39]

$$
\sigma_{\bar{q} q \rightarrow \sigma}(s)=\frac{\bar{\sigma} \Gamma}{\left(\sqrt{s}-m_{\sigma}\right)^{2}+\left(\frac{1}{2} \Gamma\right)^{2}}
$$

with a constant factor $\bar{\sigma}$ and the mass of the created unstable particle $m_{\sigma}$.

given by

$$
\Delta E=\sqrt{s}, \quad \Delta \boldsymbol{P}=\boldsymbol{p}_{1}+\boldsymbol{p}_{2} .
$$

At the particles' interaction point the particles are removed from the ensemble and their total energy and momentum are transferred to the field $\phi$ at this point, keeping the total energy and the total momentum conserved. This transfer is done by changing the field energy and momentum at the interaction point of the particles using our proposed method. The energy and momentum difference equations $(10)$ and $(11)$ are solved for the interaction-time step with a numerical solver. The field $\phi(\boldsymbol{x}, t)$ is changed by employing the 3D Gaussian parameterization $\delta \phi(\boldsymbol{x})(12)$. By changing the amplitude as well as the direction of motion of the Gaussian, both the energy and momentum can be changed within the interaction volume until $\Delta E$ and $\Delta \boldsymbol{P}$ are transferred to the field. Figure 1 shows a simplified version of this process in which small Gaussian blobs over a small volume generate small energy excitations on the field $\phi$.

To guarantee thermal and chemical equilibration, the inverse process has to be implemented according to the principle of detailed balance. This has several implications. First, the average interaction rates of particle creation and annihilation has to be the same for a given temperature, leading to no change of net-particle number. Secondly, the average energy exchange per process has to be the same for both processes. Finally, the spectra of both processes have to be the same.

We have already discussed the method for particle annihilation. The inverse process, particle production, has to be modeled differently, because the field has no initial particles which we could use for Monte-Carlo sampling of a collision term. Instead, we only have the scalar field $\phi$ and its properties like energy and momentum density, from which we have to derive particle-like properties. This step is again subject to the underlying physical model. For every point in space, we assume the field excitations to consist of unstable particles which can decay to stable particles, $\sigma \rightarrow \bar{q} q$. In case of a decay, the field $\phi$ loses the amount of energy at the interaction point, leading to an effective damping of the field. This decay process is modeled in two steps. First we have to assume a distribution function $f_{\sigma}(\boldsymbol{x}, \boldsymbol{p}, t)$ for $\sigma$ particles at every possible interaction point. The properties of $f_{\sigma}(\boldsymbol{x}, \boldsymbol{p}, t)$ have to be derived from the field properties at every point in space which is done by assuming local equilibrium via coarse graining within a field cell. To be consistent with detailed balance, the equilibrium distribution for $f_{\sigma}$ must have the same temperature as the particle's distributions, $f_{q}$ and $f_{\bar{q}}$. In the linear $\sigma$ model, the potential and therefore the equilibrium-mean field has a thermodynamical temperature dependence, which maps a mean-field value for every temperature $T \rightarrow\langle\phi\rangle(T)$. By inverting this relation $\langle\phi\rangle(T) \rightarrow T$, we can calculate the effective temperature of the field at every point of the field. The resulting phase-space distribution function, $f_{\sigma}$ defines the particle density by

$$
n_{\sigma}(\boldsymbol{x}, t)=\int \frac{\mathrm{d}^{3} \boldsymbol{p}}{(2 \pi)^{3}} f_{\sigma}(\boldsymbol{x}, \boldsymbol{p}, t) .
$$


Depending on the underlying distribution function $f_{\sigma}$ a relation between the particle and energy density has to be found

$$
n_{\sigma}(\boldsymbol{x}, t) \rightarrow n_{\sigma}(\boldsymbol{x}, t, \epsilon(\boldsymbol{x})) .
$$

The energy density $\epsilon$ is fixed by assuming the same energy density for the distribution function and the field:

$$
\epsilon=T^{00}=\int \frac{\mathrm{d}^{3} \boldsymbol{p}}{(2 \pi)^{3}} E f_{\sigma}(\boldsymbol{x}, \boldsymbol{p}, t) \equiv E(\sigma(\boldsymbol{x}), \dot{\sigma}(\boldsymbol{x}))
$$

The chosen distribution function and particle density has to be consistent with the decay process. Additionally, any momentum of the field with $\boldsymbol{p} \neq \mathbf{0}$ implies a non-zero collective velocity of the coarse-grained distribution function $f_{\sigma}$. This effect is covered by a relativistic boost of the distribution function with the mean velocity of the field via the four-velocity $u^{\mu}=p^{\mu} / E$. The field energy and momentum, $E$ and $\boldsymbol{p}$, contained in the cell around $\boldsymbol{x}$ are determined according to (5). In our calculations we have used the boosted Boltzmann distribution for the particles $q$, anti-particles $\bar{q}$ and the $\sigma$-particles,

$$
f_{\sigma} \sim \exp \left(-\frac{p_{\mu} \cdot u^{\mu}(\boldsymbol{x})}{T}\right)
$$

but the local energy relation (57) can easily be extended to other distributions like the BoseEinstein distribution. After calculating a distribution function, $\sigma$ particles are sampled from $f_{\sigma}$ with Monte-Carlo methods. For every sampled particle the decay probability is calculated. In the center of mass system of the particle, the decay probability is given by

$$
\Gamma_{\sigma}=\frac{g^{2}}{8 \pi m_{\sigma}} \sqrt{1-\frac{4 m_{q}^{2}}{m_{\sigma}^{2}}}
$$

with a $\Gamma$ consistent with (53). If a $\sigma$-particle decays, the energy and momentum of the particle is calculated with the assumption of all particles being scalar

$$
\Delta E=E_{\sigma}, \quad \Delta \boldsymbol{P}=\boldsymbol{P}_{\sigma}
$$

The resulting amount of energy and momentum is removed again from the field around the interaction point with help of the four energy and momentum difference equations (10) and (11) and the Gaussian parameterization (12). Again, this parameterization leads to a small interaction volume from which the energy is dissipated.

To come back to our notation of an interaction-probability distribution, we use the above discussed concepts to formulate an interaction probability density per numerical cell

$$
\begin{aligned}
& P(\Delta E, \Delta \boldsymbol{P}, \Delta t)= \\
& \quad \sum_{i, j}^{N_{\text {cell }}} \delta(\Delta E-\sqrt{s}) \delta\left(\Delta \boldsymbol{P}-\left(\boldsymbol{p}_{i}+\boldsymbol{p}_{j}\right)\right) \frac{\hat{\sigma}_{\bar{q} q \rightarrow \sigma} v_{\text {rel }}(s) \Delta t}{\Delta V N_{\text {test }}} \\
& \quad+\delta\left(\Delta E-E_{\sigma}\right) \delta\left(\Delta \boldsymbol{P}-\boldsymbol{P}_{\sigma}\right) \frac{\Gamma_{\sigma}\left(m_{\sigma}\right) n_{\sigma}(\phi(\boldsymbol{x}), t) \Delta t}{\Delta V} \\
& \quad+\operatorname{Pr}_{0} \delta(\Delta E) \delta(\Delta \boldsymbol{P})
\end{aligned}
$$


with the sum over all particles $N_{\text {cell }}$ in a cell.

Figs. 5- 10 show the results of the described simulation. Over the whole simulation time, the total energy and momentum stays constant, cf. Fig. 5, while the particle number and the scalar mean field show thermal fluctuations around a stable mean value. Fig. 6 shows the total particle number which fluctuates around a mean value. Field and particles are coupled via chemical processes and exchange energy and momentum, leading to fluctuations in both local energy densities and their total energy, as shown in Fig. 5. Any particle-annihilation process increases the energy of the field, any decay of field modes leads to an increase of the particle number. All fluctuations caused by these interactions lead to a strong (negative) correlation between the particle number and the mean field. Overall, the total energy of the system is conserved and shows only numerical fluctuations.

Fig. 7 represents the scalar-field distribution, showing a stable Gaussian distribution. For a thermal field this is the expected behavior, similar to the Langevin result (33). The width of the Gaussian shows small thermal fluctuations over time, additionally does the mean-value drift as a global fluctuation.

This is a remarkable result, because our scalar field shows the distribution of a thermal field which usually is archived by coupling the field to a heatbath via a Langevin-like process. A classical field, coupled to Gaussian noise, would show an equipartition of energy of $\frac{1}{2} k_{B} T$ for every degree of freedom. However, integrating over all modes leads to the famous classical ultraviolet catastrophe. In our case, the field shows a slightly different distribution of energy over the modes as shown in figure 9. For large wavelengths, the modes are populated according to the equipartition theorem; for small wavelengths the modes are strongly suppressed by the soft cutoff given by (47) and (46). The finite extension of the interaction volume leads to a smeared distribution of energy which is transferred to different modes. However, no modes with a wavelength much smaller than the extension of the interaction volume can be excited. The shape of the interaction parameterization $(12)$ is directly reflected in the spectrum of the kinetic energy, as can be seen by the analytic line in the figure. The effective extension of the interaction volume $\sigma_{\text {eff }}$ in Fig. 9 is given by

$$
\sigma_{\text {eff }}=\sqrt{\sigma_{x}^{2}+\sigma_{y}^{2}+\sigma_{z}^{2}}
$$

This soft cutoff is the first interesting feature of the proposed method. The second aspect is that, while classical Langevin models require an ad-hoc description of a stochastic force for thermalization, the proposed method does not need a non-deterministic random source. Instead, the random process is determined by a physically motivated microscopic model for the interactions of the particles and fields, having full control over the interaction rate as well as the energy and momentum exchange. Fig. 8 shows an initially vanishing scalar field with some field excitations generated by particle interactions. These particle-annihilation processes increase the field's energy, and large field modes are created. After some time, these modes overlap and the field will start to show a random, Gaussian distribution. Fig. 7 shows the field's distribution after thermalization. The distribution function of the particles show a thermal Boltzmann distribution $f_{q}(E) \sim \exp (-E / T)$ as shown in Fig. 10. The temperature of the particles is the same as the one of the thermal field fluctuations, demonstrating the accurate implementation of the principle of detailed balance in our numerical simulation. 


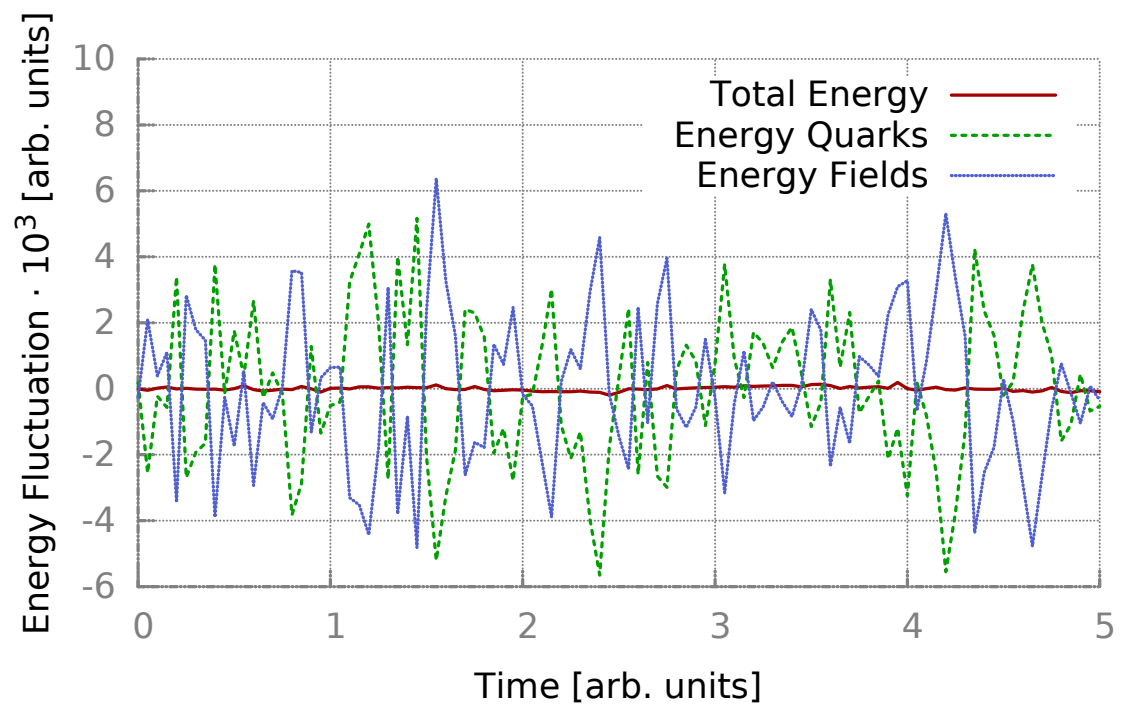

FIG. 5. (Color online) Energy fluctuations of the scalar field and the particles, $E(t)-\langle E\rangle$. Field and particles exchange energy by particle production and annihilation processes. While the total energy of the system is conserved and shows only numerical fluctuations, the energy of the components show thermal fluctuations, which are anticorrelated due to total-energy conservation. The relative fluctuations of the field's energy is $\sim 10^{-2}$, of the quarks $\sim 10^{-3}$. The total energy fluctuates on a scale of $|\Delta E| /\langle E\rangle \lesssim 5 \cdot 10^{-5}$.

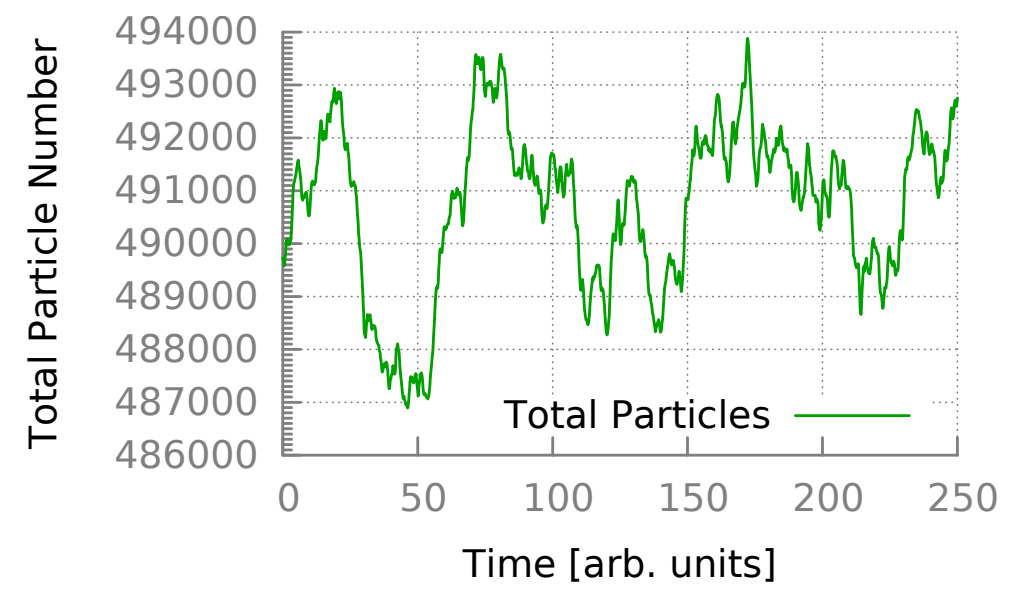

FIG. 6. (Color online) Total particle number in the thermal-box simulation. Particles can annihilate, their momentum and energy is transferred to the scalar field in form of scalar-field excitations. Because of the dynamic nature of this process, the total particle number fluctuates around the average thermal value.

\section{POSSIBLE APPLICATIONS}

We have applied the particle-field method to a transport simulation for a model with scalar fields and particles. This method is now applied to a linear $\sigma$-model [14] with the goal to investigate the dynamics of the model near and at the phase transition, where fluctuations are mainly driven by particle-field interactions. Additionally the impact of observables of the order of the phase transition is investigated, results of this study will be presented in a 


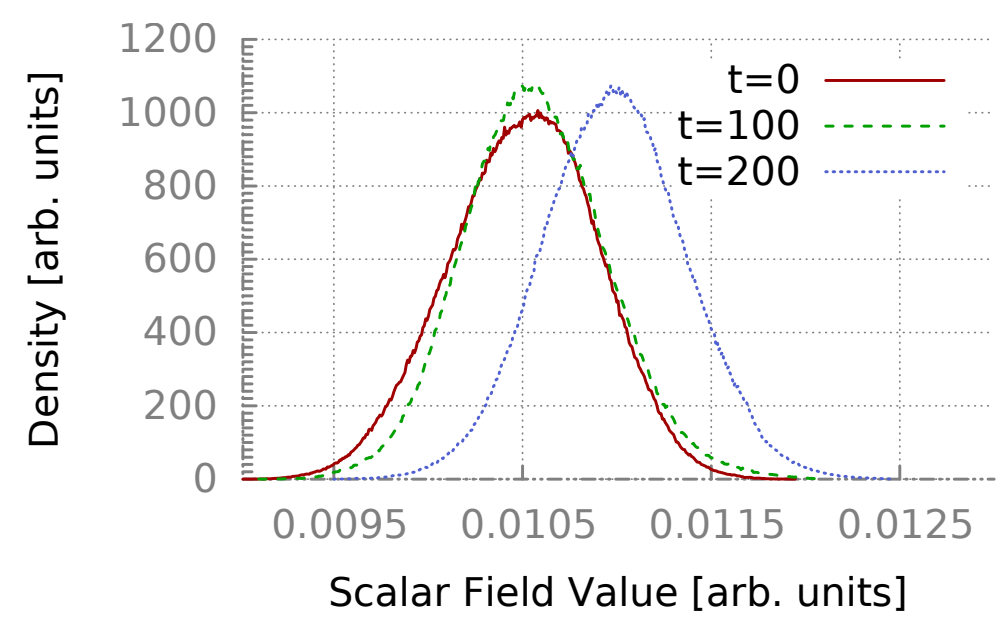

FIG. 7. (Color online) Distribution of the scalar field, showing the expected thermal Gaussdistributed fluctuations. The mean of the Gaussian can drift slowly with time due to fluctuations of the scalar mean field. Particle annihilation increases the local fluctuations of the scalar field, and particle production damps them by dissipating energy from the interaction region.

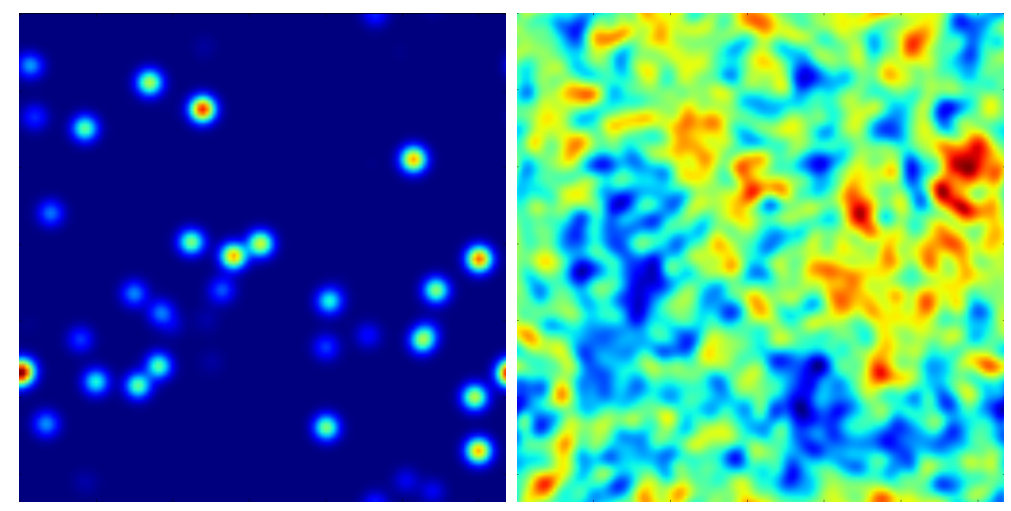

FIG. 8. (Color online) Plot of the 3D field with a cut in the $x-y$ plane at different times in the simulation. The simulation starts with a uniform scalar field without any excited modes. Due to particle creation and annihilation, field fluctuations are created dynamically within the simulation. The color coding shows the value of the scalar field. Left: Some particles have annihilated and have created small, local excitations of the scalar field in form of moving, Gaussian shaped blobs. Right: The equilibrated field in the long-time limit: Due to the particle-field interactions, Gaussian fluctuations are dynamically created by microscopic interactions.

forthcoming paper [40]. Figure 11 shows the expansion of a hot quark-droplet, which is a simple non-equilibrium setup for a heavy-ion collision. The Yukawa-coupling of the quarks to the chiral $\sigma$-field determine their effective mass, while the expansion and interactions drive fluctuations of the system. The coupling strength determines the order of the phase transition, which has a strong impact on the particle interactions, production and type of fluctuations. Results of this study will be presented in a forthcoming paper [40].

The method can easily be applied to other transport problems in heavy-ion and nuclear physics, for example in heavy-quark simulations with a hydrodynamical background [41]. Besides the already shown examples, the particle-field method can be employed whenever 

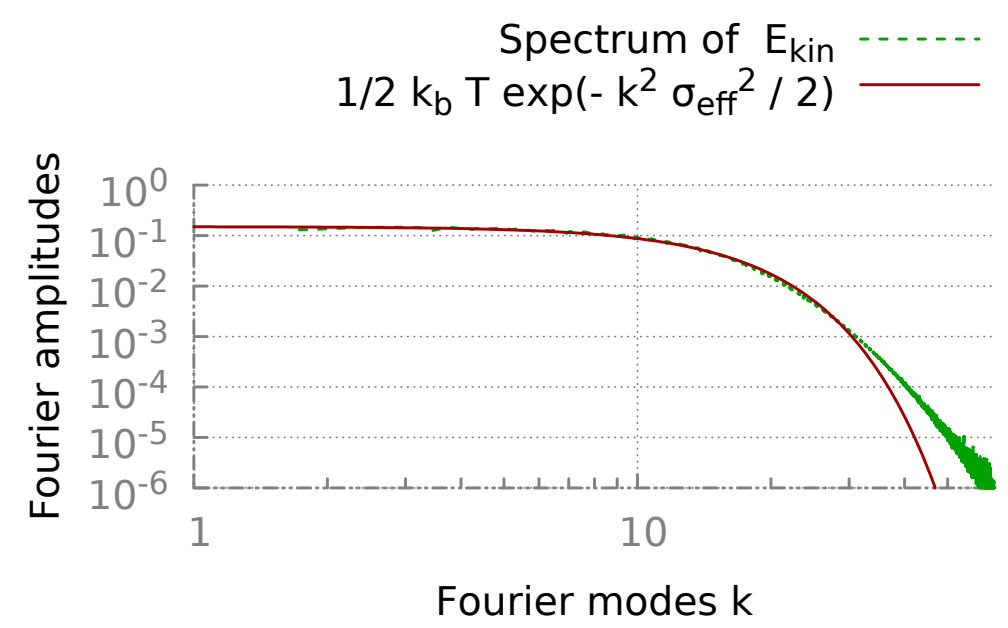

FIG. 9. (Color online) Spectrum of the field's kinetic energy $\dot{\sigma}^{2} / 2$ : While a thermal spectrum of a classical field has an average energy of $k T$ for every mode due to the equipartion theorem, with $T=0.15$ arb.units, the simulated field has an evenly distributed energy per mode only for large wavelengths. For small wavelengths, the spectrum is suppressed by a soft cut-off. This cut-off is due to the finte spatial extent of the interactions on the field. The deviation from the analytical result at higher modes can be explained by the non-linear potential in the field equations of motion.

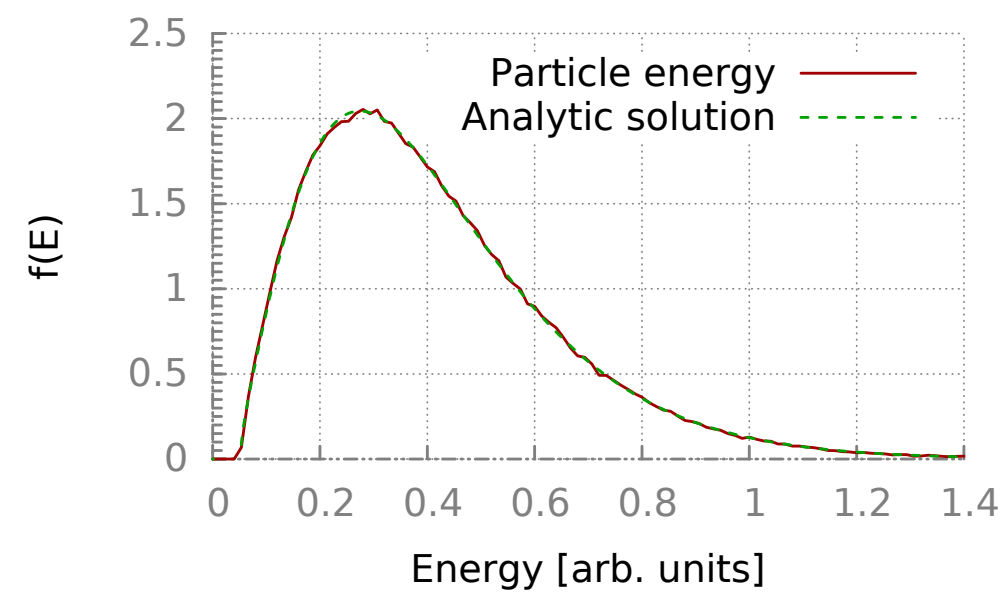

FIG. 10. (Color online) Distribution function of the particle energies showing the expected thermal Boltzmann distribution $f_{q}(E) \sim \exp (-E / T)$. By microscopic interactions particles and field can exchange energy and momentum and equilibrate to the same temperature $T=0.15 \mathrm{arb}$. units via annihilation and creation processes, demonstrating the proper implementation of the principle of detailed balance in our simulation.

noncontinuous interactions between particles and fields or fields of different types have to be modeled.

In cosmology, particle creation within the inflationary phase of the big bang are modeled by the dissipative part of an expanding scalar field $\dot{\phi}$ [42, 43]. Our method can be used for numerical simulation of the initial fluctuations on large scales. Another application in astrophysics could be to simulate pair production in electric and magnetic fields near pulsars [44] or to investigate the Sunyaev-Zel'dovich effect induced by inverse Compton scattering [45]. 


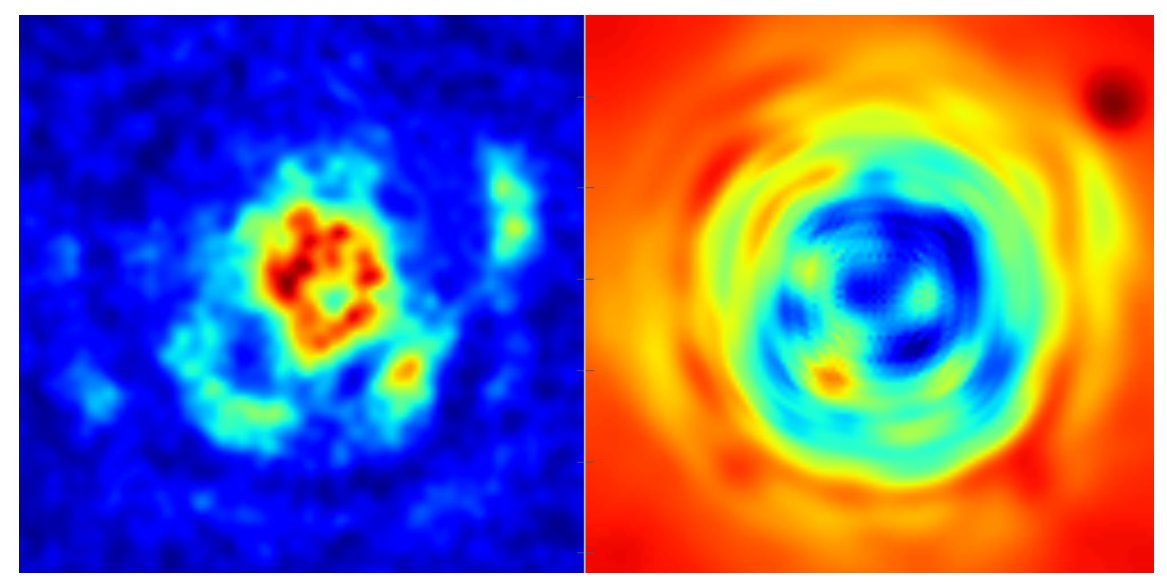

FIG. 11. (Color online) Rapid expansion of a hot quark-droplet as a simply non-equilibrium setup for a heavy-ion collision. The interaction between quarks and fields are implemented with the method purposed in this paper. The chiral $\sigma$-field can remit latent energy, which is freed by the phase transition, by creating particles. Results of this study will be presented in an upcoming paper. Left: quark density; Right: mean field value of the $\sigma$-field.

The simulated Compton effect plays also an important role in plasma physics, where damping of electromagnetic waves is affected by this process induced by density fluctuations [46]. In geophysics earthquake crack- and wave-propagation are modeled with partial differential equations [47, 48]. Our method could be used to simulate local instabilities, which deploy energy within the earthquake.

\section{SUMMARY}

In this paper we have discussed various methods to simulate interactions between particles and fields. Most of these methods are plagued by the problem to allow only continuous changes of the fields, which are hard to implement on the particle side or have some drawbacks like a non-consistent physical interpretation or create an artificial interaction-time scale. Additionally, methods like the Langevin equation are effective theories which integrate out microscopic interactions, or energy and momentum are not conserved.

In this paper we have presented a new method, which allows to describe and simulate noncontinuous interactions with exact energy and momentum conservation at all times. The position, strength and time of the interactions can be derived microscopically and simulated with Monte-Carlo methods. To give examples for this method, we have applied this method to a simple harmonic oscillator which dissipates energy by losing discrete energy quanta. In a second example the harmonic oscillator is additionally coupled to a stochastic force, the overall system behaves like an oscillator coupled to white-noise fluctuating forces as in a classical Langevin-equation. This example is generalized to a $(1+1) \mathrm{D}$ scalar field. All three systems behave in very good agreement with the analytic expectations. In the last and most complex example a $(3+1) \mathrm{D}$ scalar field is coupled to an ensemble of particles and anti-particles. Excitations of the scalar field are treated as particles which can decay via microscopic interactions and create new particle-anti-particle pairs. Additionally particles can annihilate and create field quanta. By obeying the principle of detailed balance both particles and fields reach thermal equilibrium with the same temperature, fluctuations on the 
field are generated dynamically by field-particle interactions and have not to be implemented via ad-hoc assumptions like Gaussian white noise.

In an outlook several potential interesting applications of various kinds of simulations have been suggested.

\section{ACKNOWLEDGMENTS}

This work was partially supported by the Bundesministerium für Bildung und Forschung (BMBF Förderkennzeichen 05P12RFFTS) and by the Helmholtz International Center for FAIR (HIC for FAIR) within the framework of the LOEWE program (Landesoffensive zur Entwicklung Wissenschaftlich-Ökonomischer Exzellenz) launched by the State of Hesse. C. W. and A. M. acknowledge support by the Helmholtz Graduate School for Hadron and Ion Research (HGS-HIRe), and the Helmholtz Research School for Quark Matter Studies in Heavy Ion Collisions (HQM). Numerical computations have been performed at the Center for Scientific Computing (CSC). H. v. H. has been supported by the Deutsche Forschungsgemeinschaft (DFG) under grant number GR 1536/8-1. C.W. has been supported by BMBF under grant number 0512RFFTS.

\section{Appendix A: The principle of detailed balance for particle decay and recombination}

In this appendix we briefly summarize our treatment of the particle-annihilation process and the corresponding inverse decay process with regard to the principle of detailed balance. To keep the discussion at the most simple level possible, we consider a purely bosonic model. The relevant interaction part of the Lagrangian reads

$$
\mathcal{L}_{\mathrm{I}}=\lambda \sigma q^{*} q
$$

where $\sigma$, describing $\sigma$ particles of mass $m_{\sigma}$, is a real and $q$ a complex scalar field, describing particles with mass $2 m<m_{\sigma}$. At tree level the matrix elements for decay and recombination are the same and simply given by

$$
\mathcal{M}_{\sigma \rightarrow q \bar{q}}=\sum_{\sigma} \underbrace{\bar{q}}_{\bar{q}}=-\mathrm{i} \lambda=\mathcal{M}
$$

Assuming net- $q$-particle neutrality $\left(f_{q}=f_{\bar{q}}\right)$, the corresponding Boltzmann-Vlasov equations for $q$ and $\sigma$ particles thus read

$$
\begin{array}{r}
\partial_{t} f_{q}+\frac{\boldsymbol{p}}{E_{q}} \cdot \frac{\partial f_{q}}{\partial \boldsymbol{x}}+\boldsymbol{F}(\boldsymbol{x}, \boldsymbol{p}, t) \frac{\partial f_{q}}{\partial \boldsymbol{p}}=\mathcal{C}_{q}\left[f_{q}, f_{\sigma}\right] \\
\partial_{t} f_{\sigma}+\frac{\boldsymbol{q}}{E_{\sigma}} \cdot \frac{\partial f_{\sigma}}{\partial \boldsymbol{x}}=\mathcal{C}_{\sigma}\left[f_{q}, f_{\sigma}\right]
\end{array}
$$


The collision terms are given by the transition rates for creation and annihilation of $q$ and $\sigma$ particles,

$$
\begin{aligned}
& \mathcal{C}_{q}=\frac{\lambda^{2}}{2 E_{q}} \int_{\mathbb{R}^{3}} \frac{\mathrm{d}^{3} \boldsymbol{p}_{2}}{(2 \pi)^{3} 2 E_{q 2}} \int_{\mathbb{R}^{3}} \frac{\mathrm{d}^{3} \boldsymbol{q}}{(2 \pi)^{3} 2 E_{\sigma}} \\
&(2 \pi)^{4} \delta^{(4)}\left(p_{1}+p_{2}-q\right) \\
& {\left[f_{\sigma}(\boldsymbol{x}, \boldsymbol{q}, t)-f_{q}(\boldsymbol{x}, \boldsymbol{p}, t) f_{\bar{q}}\left(\boldsymbol{x}, \boldsymbol{p}_{2}, t\right)\right], } \\
& \mathcal{C}_{\sigma}=\frac{\lambda^{2}}{2 E_{\sigma}} \int_{\mathbb{R}^{3}} \frac{\mathrm{d}^{3} \boldsymbol{p}}{(2 \pi)^{3} 2 E_{q}} \int_{\mathbb{R}^{3}} \frac{\mathrm{d}^{3} \boldsymbol{p}_{2}}{(2 \pi)^{3} 2 E_{q 2}} \\
&(2 \pi)^{4} \delta^{(4)}\left(p_{1}+p_{2}-q\right) \\
& {\left[f_{q}(\boldsymbol{x}, \boldsymbol{p}, t) f_{\bar{q}}\left(\boldsymbol{x}, \boldsymbol{p}_{2}, t\right)-f_{\sigma}(\boldsymbol{x}, \boldsymbol{q}, t)\right] . }
\end{aligned}
$$

These collision terms obviously fulfill the principle of detailed balance. Due to Boltzmann's $\mathrm{H}$ theorem, (local) equilibrium is reached when the square brackets in the above integrals vanish, and this is indeed the case for the Boltzmann distributions,

$$
f_{q}(p)=\exp (-p \cdot u / T), \quad f_{\sigma}(q)=\exp (-q \cdot u / T),
$$

where $u=u(t, \boldsymbol{x})$ is the four-velocity flow field, and $T=T(t, \boldsymbol{x})$ the temperature field [49]. Further, the four-momenta of all particles in (A5) and (A6) are assumed to fulfill the corresponding on-shell conditions, i.e., $E_{q}=\sqrt{\boldsymbol{p}^{2}+m_{q}^{2}}$ and $E_{\sigma}=\sqrt{\boldsymbol{q}^{2}+m_{\sigma}^{2}}$. This causes the difficulty that the recombination process (A3) is ineffective due to the on-shell constraint $\left(p+p_{2}\right)^{2}=m_{\sigma}^{2}$, which is artificial since an unstable particle has a finite-width spectral function. Thus, using the width according to the tree-level matrix element (A3),

$$
\Gamma_{\sigma}=\frac{\lambda^{2}}{8 \pi m_{\sigma}} \sqrt{1-\frac{4 m_{q}^{2}}{m_{\sigma}^{2}}},
$$

we substitute in $\mathrm{A} 5$ the integration operator

$$
\int_{\mathbb{R}^{3}} \frac{\mathrm{d}^{3} \boldsymbol{q}}{(2 \pi)^{3} 2 E_{\sigma}}=\int_{\mathbb{R}^{4}} \frac{\mathrm{d}^{4} q}{(2 \pi)^{4}} \Theta\left(q^{0}\right) \delta\left[\left(q^{0}\right)^{2}-E_{\sigma}^{2}\right]
$$

by

$$
\int_{\mathbb{R}^{4}} \frac{\mathrm{d}^{4} q}{(2 \pi)^{4}} \frac{\Gamma}{\left(q_{0}-E_{\sigma}\right)^{2}+\Gamma_{\sigma}^{2} / 4}
$$

For consistency and to preserve detailed balance, also (A6) has to be averaged by the BreitWigner distribution in the same way, i.e., we have to substitute

$$
\delta\left(q^{0}-p_{1}^{0}-p_{2}^{0}\right) \rightarrow \frac{1}{2 \pi} \frac{\Gamma}{\left(p_{1}^{0}+p_{2}^{0}-E_{\sigma}\right)^{2}+\Gamma_{\sigma}^{2} / 4}
$$

for the energy-conserving $\delta$ distribution in the collision term. A fully self-consistent treatment of particles of finite width within a kinetic approach [50, 51] is beyond the scope of this paper.

[1] L. L. Carter and E. D. Cashwell, Particle-transport simulation with the Monte Carlo method, Tech. Rep. (Los Alamos Scientific Lab., N. Mex.(USA), 1975). 
[2] H. J. Rothe, Lattice gauge theories (World Scientific, 2012).

[3] B. Friman, C. Höhne, J. Knoll, S. Leupold, J. Randrup, and P. S. R. Rapp, The CBM Physics Book: Compressed Baryonic Matter in Laboratory Experiments, Lecture Notes in Physics (Springer, 2011).

[4] Z. Xu and C. Greiner, Physical Review C 71 (2005), 10.1103/physrevc.71.064901.

[5] D. L. Ermak and J. McCammon, The Journal of chemical physics 69, 1352 (1978).

[6] M. Fixman, Macromolecules 19, 1204 (1986).

[7] L. E. Reichl and I. Prigogine, A modern course in statistical physics, Vol. 186 (University of Texas press Austin, 1980).

[8] A. A. Vlasov, Soviet Physics Uspekhi 10, 721 (1968).

[9] T. Dupree, Physics of Fluids (1958-1988) 9, 1773 (1966).

[10] C. K. Birdsall and A. B. Langdon, Plasma physics via computer simulation (CRC Press, 2004).

[11] M. Gell-Mann and M. Lvy, Il Nuovo Cimento 16, 705 (1960).

[12] J. Berges and J. Cox, Physics Letters B 517, 369 (2001).

[13] F. Cooper, S. Habib, Y. Kluger, and E. Mottola, Phys. Rev. D 55, 6471 (1997).

[14] C. Wesp, Dynamical simulation of a linear sigma model (PhD Thesis), (in preperation), Institut für theoretische Physik Goethe Universtät Frankfurt (2014).

[15] G. Aarts and J. Berges, Physical Review D 64 (2001), 10.1103/physrevd.64.105010.

[16] J. Berges, S. Borsányi, and J. Serreau, Nuclear Physics B 660, 51 (2003)

[17] S. Juchem, W. Cassing, and C. Greiner, Phys. Rev. D 69, 025006 (2004)

[18] D. S. Lemons and A. Gythiel, American Journal of Physics 65, 1079 (1997),

[19] A. Bonasera, F. Gulminelli, and J. Molitoris, Physics Reports 243, 1 (1994).

[20] J. Randrup and B. Remaud, Nuclear Physics A 514, 339 (1990).

[21] P. Chomaz, G. Burgio, and J. Randrup, Physics Letters B 254, 340 (1991).

[22] C. Greiner and S. Leupold, Annals of Physics 270, 328 (1998).

[23] C. Greiner and B. Müller, Phys. Rev. D 55, 1026 (1997).

[24] T. S. Biró and C. Greiner, Phys. Rev. Lett. 79, 3138 (1997).

[25] Z. Xu and C. Greiner, Physical Review D 62 (2000), 10.1103/physrevd.62.036012.

[26] D. H. Rischke, Phys. Rev. C 58, 2331 (1998).

[27] M. Nahrgang, S. Leupold, and M. Bleicher, Physics Letters B 711, 109 (2012).

[28] M. Nahrgang, S. Leupold, C. Herold, and M. Bleicher, Physical Review C 84 (2011), 10.1103/physrevc.84.024912.

[29] C. Herold, M. Nahrgang, I. Mishustin, and M. Bleicher, Physical Review C 87, 014907 (2013).

[30] J. Schmidt, A. Meistrenko, H. van Hees, Z. Xu, and C. Greiner, Physical Review E 91 (2015), 10.1103/physreve.91.032125.

[31] Li, Numerical studies of the Klein-Gordon-Schrödinger equations, Master's thesis, Sichuan University (2006).

[32] G. B. Whitham, Linear and nonlinear waves, Vol. 42 (John Wiley \& Sons, 2011).

[33] L. Kofman, A. Linde, and A. A. Starobinsky, Phys. Rev. Lett. 73, 3195 (1994).

[34] R. Feynman and F. Vernon, Annals of Physics 24, 118 (1963),

[35] M. Toda, R. Kubo, N. Sait, and N. Hashitsume, Statistical Physics II: Nonequilibrium Statistical Mechanics, Series C, English Authors (Springer Berlin Heidelberg, 1991).

[36] T. Lindstrom, Bulletin of the London Mathematical Society 25, 83 (1993),

[37] N. Wiener, Acta Mathematica 55, 117 (1930).

[38] G. Breit and E. Wigner, Phys. Rev. 49, 519 (1936).

[39] A. R. Bohm and Y. Sato, Phys. Rev. D 71, 085018 (2005). 
[40] C. Wesp et al., in preperation (2015).

[41] Y. Akamatsu, T. Hatsuda, and T. Hirano, Phys. Rev. C 79, 054907 (2009).

[42] L. Abbott, E. Farhi, and M. B. Wise, Physics Letters B 117, 29 (1982).

[43] D. Boyanovsky, H. J. de Vega, R. Holman, D.-S. Lee, and A. Singh, Phys. Rev. D 51, 4419 (1995).

[44] J. K. Daugherty and I. Lerche, Phys. Rev. D 14, 340 (1976).

[45] S. M. Molnar and M. Birkinshaw, ApJ 523, 78 (1999).

[46] W. P. Leemans, C. E. Clayton, W. B. Mori, K. A. Marsh, P. K. Kaw, A. Dyson, C. Joshi, and J. M. Wallace, Phys. Rev. A 46, 1091 (1992).

[47] T. MIYATAKE, Journal of Physics of the Earth 28, 565 (1980).

[48] H. Bao, J. Bielak, O. Ghattas, L. F. Kallivokas, D. R. O'Hallaron, J. R. Shewchuk, and J. Xu, Computer Methods in Applied Mechanics and Engineering 152, 85 (1998), containing papers presented at the Symposium on Advances in Computational Mechanics.

[49] C. Cercignani and G. M. Kremer, The relativistic Boltzmann Equation: Theory and Applications (Springer, Basel, 2002).

[50] Y. B. Ivanov, J. Knoll, and D. N. Voskresensky, Nucl. Phys. A 657, 413 (1999).

[51] Y. B. Ivanov, J. Knoll, and D. Voskresensky, Nucl. Phys. A 672, 313 (2000). 